\title{
Diminishing returns as a function of disturbance
}

\author{
Kimmo Sorjonen ${ }^{\text {Corresp., }}{ }^{\text {, Bo }}$ Melin ${ }^{1}$ \\ ${ }^{1}$ Department of Clinical Neuroscience, Karolinska Institutet, Stockholm, Sweden \\ Corresponding Author: Kimmo Sorjonen \\ Email address: kimmo.sorjonen@ki.se
}

According to Spearman's law of diminishing returns (SLODR), IQ test scores are more $g$ saturated among those with low, compared to those with high, ability. The present simulation shows that such difference in saturation can be observed if test scores are affected by a disturbing factor, e.g. low motivation, illness, or linguistic confusion, that varies in magnitude between individuals. More contemporary criteria of SLODR can also be satisfied if test scores are affected by disturbance, especially if the disturbance variable is negatively skewed. This indicates a possible threat against the validity of findings supporting SLODR and points at the importance for researchers to try to eliminate the influence of such disturbing factors from their studies. 
1 Title: Diminishing returns as a function of disturbance

2 Authors: Kimmo Sorjonen ${ }^{1} \&$ Bo Melin $^{1}$

$3 \quad{ }^{1}$ Department of Clinical Neuroscience, Karolinska Institutet, Stockholm, Sweden

4

5 Corresponding Author:

6 Name: Kimmo Sorjonen

7 Adress: Division of Psychology, Department of Clinical Neuroscience, Karolinska Institutet, 171

865 Solna, Sweden

9 Phone: +46-76-878 4236

Email: kimmo.sorjonen@ki.se

Abstract

According to Spearman's law of diminishing returns (SLODR), IQ test scores are more $g$ saturated among those with low, compared to those with high, ability. The present simulation shows that such difference in saturation can be observed if test scores are affected by a disturbing factor, e.g. low motivation, illness, or linguistic confusion, that varies in magnitude between individuals. More contemporary criteria of SLODR can also be satisfied if test scores are affected by disturbance, especially if the disturbance variable is negatively skewed. This indicates a possible threat against the validity of findings supporting SLODR and points at the importance for researchers to try to eliminate the influence of such disturbing factors from their

21 studies. 
23 Keywords: differentiation hypothesis; disturbance; performance; simulation; Spearman's law of

24 diminishing returns; validity 
To measure abilities can be tricky, as performances can be assumed to be affected not only

by peoples' abilities but also by various disturbing factors. That is why athletes' performances

tend to fluctuate more than their underlying true abilities can be supposed to do. Here the

disturbing factors could be such things as bad weather, a sore knee, or lack of motivation.

Performances on tests of cognitive or creative abilities, for instance, could also be influenced by disturbing factors, such as low motivation, illness, or linguistic confusion.

positive influence on creative potential but only up to a certain level, the threshold is often set at

IQ 120, after which a further increase in intelligence should have no additional effect on creative potential. This hypothesis has received some empirical support (e.g. Cho, Te Nijenhuis, Van Vianen, Kim, \& Lee, 2010; Jauk, Benedek, Dunst, \& Neubauer, 2013; Shi, Wang, Yang, Zhang, \& Xu, 2017, but see Kim, 2005, for a non-confirming meta-analysis).

However, a simulation by Sorjonen, Ingre, and Melin (2019) indicates a high probability to observe such threshold-like associations if subjects experience varying degrees of disturbance that affects their performances both on the tests of intelligence and on the tests of creativity. A high degree of disturbance will tend to attenuate the association between measured intelligence and creativity among those with high true abilities while it accentuates the association among those with low abilities. hypothesis" (Garrett, 1946), has often been evaluated, and demonstrated, with a method where the $g$ saturation of intelligence test scores, which corresponds to the strength of the correlations between the test scores, has been compared between groups assumed to have high or low average 
50 g. An often-observed lower degree of $g$ saturation in the former group has been taken to indicate

51 that intelligence is more differentiated/specialized among those with high $g$, with some

52 individuals having, for example, especially high verbal or spatial intelligence. A higher $g$

53 saturation among those with assumed low $g$, on the other hand, has been seen to suggest that in

54 this subgroup intelligence is less specialized and more one-dimensional.

55 For example, Deary et al. (1996) claimed support for SLODR after analyzing data from

56 Irish schoolchildren $(N=10,535)$ on eight Differential Aptitude Tests (DAT) subtests, measuring

57 (1) Verbal reasoning, (2) Numerical ability, (3) Abstract reasoning, (4) Clerical speed and

58 accuracy, (5) Mechanical reasoning, (6) Space relations, (7) Spelling, and (8) Language usage.

59 When dividing the participants into two subgroups, high and low scorers, based on verbal

60 reasoning, the amount of variance in the seven remaining subtests accounted for by a first

61 unrotated principal component equaled $36.2 \%$ and $43.6 \%$ among the high and low scorers,

62 respectively. When the division was based on numerical ability and space relations the

63 corresponding values were $40.9 \%$ and $46.2 \%$ for high scorers and $46.2 \%$ and $52.9 \%$ for low

64 scorers, respectively. Other studies claiming support for SLODR include Detterman and Daniel

65 (1989), Legree, Pifer, and Grafton (1996), Reynolds and Keith (2007), Tucker-Drob (2009), as

66 well as a meta-analysis by Blum and Holling (2017).

67 However, the traditional method of calculating amount of variance accounted for by a first 68 unrotated principal component, or the average intersubtest correlation, in subgroups assumed to 69 differ in $g$ has been criticized. It has, for example, been claimed that this method can give 70 confounded results possibly affected by the arbitrary decision how to form the subgroups or by

71 the skewness of test scores (Molenaar, Dolan, Wicherts, \& van der Maas, 2010; Murray, Dixon, $72 \&$ Johnson, 2013). According to more contemporary thoughts, ability differentiation, a.k.a. 
73 diminishing returns, might be operationalized as (1) a negatively skewed latent $g$, (2)

74 heteroscedastic subtest residuals, with a larger residual variance among those with high compared to low $g$, and (3) non-linear $g$ loadings (Hessen \& Dolan, 2009; Molenaar, Dolan, \& van der Maas, 2011; Molenaar, Dolan, \& Verhelst, 2010; Murray et al., 2013; Tucker-Drob, 2009). The fulfilment of these criteria can be analyzed with structural equation modelling (SEM) based methods. there really is any convincing support for SLODR. For example, when using a traditional method, Murray et al. (2013) found that support for SLODR was proportional to the average skew of the test battery, i.e. mainly found in positively skewed batteries. When using more contemporary methods, they found heteroscedastic residuals, positively correlated with $g$, in positively skewed test batteries, while the criterium of non-linear $g$-loadings, on the contrary, was found in negatively skewed batteries. The criterium of a negatively skewed latent $g$ was never fulfilled. In analyzes of data from young Spanish adults $(N=588)$ on 14 WAIS-III subtests, Molenaar et al. (2011) found some indications of quadratic second-order factor loadings and a negatively skewed second order common factor $(g)$. However, contrary to predictions by SLODR, residual variances tended to decrease with increased $g$. As mentioned above, simulations have indicated a high probability to observe thresholdlike associations, for example between intelligence and creativity, if subjects experience varying

92 degrees of disturbance that affects their performances (Sorjonen et al., 2019). Due to its

93 similarity to the intelligence-creativity threshold hypothesis, with both proposing a stronger association between performances among those with low ability, it is possible that SLODR might

95 be sensitive to the influence of disturbing factors as well. The objective of the present simulation 
96 study was to assess this possibility. Although the traditional method to analyze SLODR through

97 degree of $g$ saturation in subgroups based on different levels of $g$ has been deemed untrustworthy

98 (see above), we still include it in the present study in order to see how it might be affected by

99 disturbance in the measurement of intelligence. We do this due to personal curiosity and because

100 a majority of all studies of SLODR have used this method. They do, for example, seem to

101 constitute most of the studies in Blum and Holling's (2017) meta-analysis. However, anyone

102 wishing not to know how the traditional method is affected by disturbance is advised not to read

103 the sections with the heading "Traditional paradigm" below, nor the first paragraph in the

104 discussion, nor to look at panel A in Figure 1 and Figure 2.

Method

107

108

109

110

111

112

113

114

115

116

117

118

\section{Traditional paradigm}

Using R 3.5.0 statistical software (R Core Team, 2018) and the psych package (Revelle, 2018), data was simulated and analyzed through the following steps (panel A in Figure 1, script available at https://osf.io/7ydp8/): (1) 10,000 virtual subjects were allocated a true $g$ score from a random normal distribution $(M=100, S D=15)$; (2) The subjects were allocated 15 test scores $(M=100, S D=15)$ with a defined population correlation with the $g$ score. The correlation for the first test score, the selection variable, was set to either $0.1,0.5$, or 0.9 while the correlations for the other 14 variables were drawn from a random uniform distribution between 0.2 and 0.9 . Being continuous, these test scores could be seen to correspond to first order factor scores or subtest scores rather than to traditional dichotomous test items and, consequently, the calculated factor loadings correspond to second order factor loadings; (3) The virtual subjects were allocated a disturbance score from a random beta distribution that was either negatively (alpha $=$ 
1199 , beta $=1)$ or positively $($ alpha $=1$, beta $=9)$ skewed or approximately normally distributed

$120($ alpha $=9$, beta $=9)$. These disturbance scores varied between 0 and 1 with a lower value

121 indicating a higher degree of disturbance; (4) The virtual subjects' test scores, including the

122 selection variable, were multiplied by their disturbance score, except in the cases with no effect

123 of disturbance; (5) Based on their value on the selection variable (rescaled to the conventional $M$

$124=100, S D=15)$, the virtual subjects were divided into 10 subgroups within 20 points on the

125 selection variable. Hence, there was overlap between the subgroups, with some subjects included

126 in more than one group; (6) In each subgroup, the amount of variance in the test scores,

127 excluding the selection variable, that could be explained by a first un-rotated principal

128 component, i.e. $g$ saturation, was calculated. According to SLODR, there should be a negative

129 association between the mean on the selection variable and the degree of $g$ saturation in the 130 subgroups.

131 This procedure was repeated 20 times for each distribution of the disturbance variable and 132 when disturbance had no effect, and for the three levels of correlation between the selection 133 variable (undisturbed) and $g$. When using one of the test scores for creating the groups and then 134 not including this in the principal component analysis, we follow the procedure by Deary et al. 135 (1996).

More contemporary operationalization

138 As mentioned in the introduction, a more contemporary operationalization of SLODR 139 include the criteria (1) a negatively skewed latent $g,(2)$ heteroscedastic subtest residuals, with a 140 larger residual variance among those with high compared to low $g$, and (3) non-linear $g$ loadings.

141 Data was simulated similarly as described above, bar the selection variable, with one thousand 
142 simulations $(N=10,000$ in each) for each of the four types of disturbance variables - non-

143 existent, normally distributed, negatively, and positively skewed. Data was analyzed using the

144 lavaan (Rosseel, 2012) and semTools (Jorgensen, Pornprasertmanit, Schoemann, \& Rosseel, 145 2018) packages in $R$.

146 Initially, a one-factor confirmatory factor analysis (CFA) was applied to data (the blue part

147 in panel B in Figure 1). Using the lavPredict function in lavaan, $g$ was estimated for each virtual

148 subject and the skewness, with significance, of this $g$ was calculated with the skew function in

149 semTools. According to SLODR, $g$ should be significantly negatively skewed.

150 Employing the lavPredict function once more, the predicted score, and then the squared

151 difference between the observed and this predicted score, on each of the fifteen tests was

152 calculated for each virtual subject. The individual mean on these squared differences correspond

153 to the individual residual variance, i.e. variance in the test scores that is not accounted for by the

154 individual's $g$. According to SLODR, there should be a significant positive correlation between $g$

155 and this residual variance. Following Hessen and Dolan (2009), the logarithm of residual

156 variance was used in this analysis.

157 For the third criterium, the test scores were regressed on a quadratic latent $g$ factor in

158 addition to the linear latent $g$ factor (red part in panel B in Figure 1). The factor loadings of

159 quadratic $g$ were constrained to be the square of the corresponding linear factor loadings. In this

160 way, the quadratic $g$ factor catches the acceleration in the effect of $g$ on the test scores.

161 According to SLODR, the addition of quadratic $g$ to the model should result in significant

162 improvement of model fit. Moreover, the estimated value on the quadratic $g$ factor should

163 become increasingly more negative with an increase in $g$ (= diminishing returns), i.e. there

164 should be a significant negative correlation between $g$ and $g^{2}$. 
Results

167

168

169

170

171

172

173

174

175

176

177

178

179

180

181

182

183

184

185

186

\section{Traditional paradigm}

In accordance with SLODR, a negative association between the mean value on the selection variable in subgroups and the amount of variance in the test scores that could be explained by a first un-rotated principal component, i.e. $g$ saturation, was apparent, except when disturbance was not allowed to affect the test scores (Figure 2). When the selection variable (undisturbed) was strongly correlated with the true $g$ score (panel C) this association was accentuated, but we also see a general decrease in the amount of explained variance compared to when the correlation was weaker (panels A and B). This is expected, because in the former case the subjects are more homogeneous in their $g$ and there is less room for a common factor to explain variance in the test scores. With a positively skewed disturbance factor the selection variable also became positively skewed, with few subjects at the high end of the scale, and here we see a high degree of instability in the calculated amount of explained variance.

\section{More contemporary operationalization}

In Table 1, we see that with no disturbance affecting test scores, the probability to fulfill the criteria of a negatively skewed $g$ and a positive correlation between $g$ and residual variance are close to what can be expected given a significance level of .05. Fulfillment of the criterium of non-linear $g$ loadings, which requires both a significant improvement in model fit when adding a quadratic $g$ factor to the model and a significant negative correlation between $g$ and $g^{2}$ (see the Method section), is less probable. 
With a negatively skewed disturbance variable affecting test scores, all of the

188

189

190

191

192 contemporary criteria are fulfilled in $81 \%$ of the cases, and without a requirement for a negative correlation between $g$ and $g^{2}$ we would see virtually perfect fulfillment. With a normally distributed or a positively skewed disturbance variable, the probability for a positive correlation between $g$ and residual variance and for a negative correlation between $g$ and $g^{2}$ are extremely high. However, here we always see, contrary to SLODR criteria, a positively skewed $g$ (Table 1). The effect of a negatively skewed disturbance variable is illustrated in Figure 3. In this example, true $g$ is perfectly normally distributed (skewness $=-0.001, p=0.982$, panel A) and has a weak positive linear association with the true score on one of the fifteen tests $(r=0.131, p<$ 0.001, panel B). However, if the test scores are affected by a negatively skewed (skewness = $1.521, p<0.001)$ disturbance variable, the estimated $g$ becomes negatively skewed (skewness $=$ $0.365, p<0.001$, panel C). Now we also see slightly heteroscedastic residuals that increase with estimated $g$ (correlation between $g$ and the logarithm of squared deviations from the linear prediction line in panel $\mathrm{D}=0.036, p<0.001$, note that the correlation between $g$ and the logarithm of individual residual variance over all fifteen test scores is substantially stronger, $r=$ $0.218, p<0.001)$ and a quadratic association between estimated $g$ and observed test score $(B=$ 1.266 and $p<0.001$ for the quadratic regression term, panel D).

\section{Discussion}

The present simulation shows that if test scores are affected by disturbance - which could be, for example, low motivation, illness, or linguistic confusion - that vary in magnitude between individuals, we will, similarly as studies using a traditional paradigm to assess SLODR, tend to see a higher degree of factor saturation among those with a low score on an associated selection 
210 variable compared to those with a high score. This is true irrespective of the distribution of the

211 disturbance variable. Consequently, the present findings could be added to the growing number

212 of warnings that a positive result when using the traditional paradigm cannot be trusted to

213 indicate a true SLODR-effect.

214 When evaluating the effect of disturbance using more contemporary criteria, results in

215 accordance with SLODR were seen mainly with a negatively skewed disturbance variable. With

216 a normally distributed or a positively skewed disturbance variable, two of three criteria, namely

217 heteroscedastic residual variance and quadratic factor loadings, were usually fulfilled, while the

218 skewness of estimated $g$ was in the opposite direction compared to SLODR criteria. Hence, one

219 of the advantages of these contemporary criteria seems to be that one of them, negative skewness

220 of estimated $g$, offers protection against type 1-errors, unless test scores have been affected by

221 negatively skewed disturbance, while the traditional method seems to deliver false support for

222 SLODR irrespective of the distribution of the disturbance variable. However, negatively skewed

223 disturbance would mean that most subjects receive test scores close to their true values while a

224 few perform well below their true ability, and we believe that such lack of validity among a

225 minority of participants can be quite realistic in many test situations.

226

According to Murray et al. (2013), the empirical support for SLODR can be called into

227 question as it is based mainly on studies employing traditional paradigms, which cannot

228 discriminate between a true SLODR-effect and effects of subtest characteristics, e.g. skewness.

229 The present simulation throws some additional pessimism into the pot. If using more

230 contemporary methods and finding support for SLODR in the form of a negatively skewed latent

$231 g$, heteroscedastic residuals, and quadratic factor loadings, one cannot be sure if this is due to a 
232 true SLODR-effect or the influence of some negatively skewed disturbance factor that varies in

233 degree between study participants and that affects their test scores.

234 We do not, of course, claim to have proven that all findings in accordance with SLODR are

235 due to the influence of negatively skewed disturbance. We merely point at the possibility that

236 this could have given SLODR a helping hand in some studies. This points at the importance for

237 researchers to try to eliminate, as far as possible, the influence of all such disturbing factors from

238 their studies. However, we can probably never be absolutely sure that we are measuring all of the

239 participants' true abilities rather than merely their performances.

240 Still, even if findings that support SLODR would be due to the influence of disturbance,

241 this does not necessarily mean that SLODR is just an artifact. For example, according to

242 Detterman's (1993) explanation of SLODR, as well as the more recent process-overlap theory

243 (POT, Kovacs \& Conway, 2016, 2019), the "disturbing factor" could be a deficit in some

244 important general cognitive process which restrains the functioning of the whole system.

245 Without this deficit, test scores are mainly due to more specific processes and, consequently, less

246 correlated and $g$ saturated. If Detterman's hypothesis and POT are correct, and if studies using

247 more contemporary criteria start supporting SLODR (it does not look very promising so far), one

248 of the contributions of the present simulation would be to predict that most people experience

249 only low degrees of this deficit while a few experiences a very high level.

250

251 Conclusions

252 The present simulation indicates that findings that falsely seem to support Spearman's law 253 of diminishing returns (SLODR) can be obtained if test scores are affected by a disturbance 
254 variable that varies in magnitude between respondents. This is true for contemporary

255 operationalizations of SLODR and even more so if using a traditional paradigm to analyze data. 256

\section{References}

258

259

260

261

262

263

264

265

266

267

268

269

270

271

272

273

274

275

276

Blum, D., \& Holling, H. (2017). Spearman's law of diminishing returns. A meta-analysis. Intelligence, 65, 60-66. https://doi.org/10.1016/j.intell.2017.07.004

Cho, S. H., Te Nijenhuis, J., Van Vianen, A. E. M., Kim, H. B., \& Lee, K. H. (2010). The relationship between diverse components of intelligence and creativity. Journal of Creative Behavior, 44(2), 125-137. https://doi.org/10.1002/j.2162-6057.2010.tb01329.x

Deary, I. J., Egan, V., Gibson, G. J., Austin, E. J., Brand, C. R., \& Kellaghan, T. (1996). Intelligence and the differentiation hypothesis. Intelligence, 23(2), 105-132. https://doi.org/10.1016/S0160-2896(96)90008-2

Detterman, D. K. (1993). Giftedness and intelligence: One and the same? In G. R. Bock \& K. Ackrill (Eds.), Ciba Foundation Symposium 178: The Origins and Development of High Ability (pp. 22-43). Chichester: John Wiley \& Sons.

Detterman, D. K., \& Daniel, M. H. (1989). Correlations of mental tests with each other and with cognitive variables are highest for low IQ groups. Intelligence, 13(4), 349-359. https://doi.org/10.1016/S0160-2896(89)80007-8

Garrett, H. E. (1946). A developmental theory of intelligence. The American Psychologist, 1(9), 372-378. https://doi.org/10.1037/h0056380

Hessen, D. J., \& Dolan, C. V. (2009). Heteroscedastic one-factor models and marginal maximum likelihood estimation. British Journal of Mathematical and Statistical Psychology, 62(1), 57-77. https://doi.org/10.1348/000711007X248884 
277 Jauk, E., Benedek, M., Dunst, B., \& Neubauer, A. C. (2013). The relationship between

278 intelligence and creativity: New support for the threshold hypothesis by means of empirical

279 breakpoint detection. Intelligence, 41(4), 212-221.

280 https://doi.org/10.1016/j.intell.2013.03.003

281

282

283

284

285

286

287

288

289

290

291

292

293

294

295

296

297

298

299

Jorgensen, T. D., Pornprasertmanit, S., Schoemann, A. M., \& Rosseel, Y. (2018). semTools: Useful tools for structural equation modeling. R package version 0.5-1. Retrieved from https://CRAN.R-project.org/package=semTools

Kim, K. H. (2005). Can Only Intelligent People Be Creative? Journal of Secondary Gifted Education, 16(2), 57-66.

Kovacs, K., \& Conway, A. R. A. (2016). Process overlap theory: A unified account of the general factor of intelligence. Psychological Inquiry, 27, 151-177.

Kovacs, K. \& Conway, A. R. A. (2019). What is IQ? Life beyond general intelligence. Current Directions in Psychological Science, 28, 189-194.

Legree, P. J., Pifer, M. E., \& Grafton, F. C. (1996). Correlations among cognitive abilities are lower for higher ability groups. Intelligence, 23(1), 45-57. https://doi.org/10.1016/S01602896(96)80005-5

Molenaar, D., Dolan, C. V., \& van der Maas, H. L. J. (2011). Modeling ability differentiation in the second-order factor model. Structural Equation Modeling, 18(4), 578-594. https://doi.org/10.1080/10705511.2011.607095

Molenaar, D., Dolan, C. V., \& Verhelst, N. D. (2010). Testing and modelling non-normality within the one-factor model. British Journal of Mathematical and Statistical Psychology, 63(2), 293-317. https://doi.org/10.1348/000711009X456935

Molenaar, D., Dolan, C. V., Wicherts, J. M., \& van der Maas, H. L. J. (2010). Modeling 
300

301

302

303

304

305

306

307

308

309

310

311

312

313

314

315

316

317

318

319

320

321

322

differentiation of cognitive abilities within the higher-order factor model using moderated factor analysis. Intelligence, 38(6), 611-624. https://doi.org/10.1016/j.intell.2010.09.002

Murray, A. L., Dixon, H., \& Johnson, W. (2013). Spearman's law of diminishing returns: A statistical artifact? Intelligence, 41(5), 439-451. https://doi.org/10.1016/j.intell.2013.06.007

R Core Team. (2018). R: A language and environment for statistical computing. R Foundation for Statistical Computing, Vienna, Austria. URL https:/www.R-project.org/.

Revelle, W. (2018). psych: Procedures for personality and psychological research, Northwestern University, Evanston, Illinois, USA, https:/CRAN.R-project.org/package=psych Version = 1.8.3.

Reynolds, M. R., \& Keith, T. Z. (2007). Spearman's law of diminishing returns in hierarchical models of intelligence for children and adolescents. Intelligence, 35(3), 267-281. https://doi.org/10.1016/j.intell.2006.08.002

Rosseel, Y. (2012). lavaan: An R package for structural equation modeling. Journal of Statistical Software, 48(2), 1-36. URL http://www.jstatsoft.org/v48/i02/.

Shi, B., Wang, L., Yang, J., Zhang, M., \& Xu, L. (2017). Relationship between divergent thinking and intelligence: An empirical study of the threshold hypothesis with Chinese children. Frontiers in Psychology, 8(FEB), 1-9. https://doi.org/10.3389/fpsyg.2017.00254

Sorjonen, K., Ingre, M., \& Melin, B. (2019). Threshold-like associations as a function of disturbance. PeerJ, 7, e7891. DOI 10.7717/peerj.7891

Spearman, C. (1927). The abilities of man. London: Macmillan.

Tucker-Drob, E. M. (2009). Differentiation of cognitive abilities across the life span. Developmental Psychology, 45(4), 1097-1118. https://doi.org/10.1037/a0015864 
326 Figure 1. (A) Steps in the simulation (solid lines) and analyzed parameters (dashed lines) of the

327 traditional paradigm. Test scores and a selection variable (sv) are affected by true $g(\operatorname{tg})$ but the

328 effect is attenuated by disturbance that varies in magnitude between individuals. Amount of

329 variance in the test scores explained by the first un-rotated principal component (ev) is calculated

330 separately in the subgroups defined by the selection variable. Note: 14 test scores, besides the

331 selection variable, were used in the actual simulation. (B) Illustration of the analyses when

332 evaluating more contemporary criteria of SLODR. Test scores are regressed on either one single

333 common $g$ factor (blue part) or on one linear and one quadratic $g$ factor (blue + red part). In the

334 latter case, factor loadings for the quadratic factor are constrained to equal the square of the

335 corresponding linear factor loadings. Note: 15 test scores were used in the actual simulation.

336

337 Figure 2. Amount of variance in 14 tests explained by the first un-rotated principal component

338 as a function of the mean value on the selection variable in the subgroups when the disturbance

339 variable is either negatively, normally, or positively distributed or if there is no effect of

340 disturbance on test scores. Separately for three different degrees of correlation between $g$ and the

341 selection variable (before disturbance), namely (A) 0.1, (B) 0.5, and (C) 0.9. The subgroups

342 always have a maximum range of 20 points on the selection variable. 20 simulations (adjoined

343 by a line) for each distribution of the disturbance variable in each panel and with $N=10,000$ in

344 each.

345

346 Figure 3. Illustration of the effect of a negatively skewed disturbance variable. (A) A perfectly

347 normally distributed true $g$ has (B) a weak linear association with the true score on one of the 
348 fifteen tests. When test scores are affected by a negatively skewed disturbance variable, we

349 observe (C) a negatively skewed estimated $g$ that has (D) a quadratic association with the

350 observed test score. We also observe heteroscedastic residuals that increase with increased $g$ (D).

$351 \quad N=10,000$ in each panel, black line $=$ best fitting linear association, red line $=$ best fitting

352 quadratic association.

353 


\section{Figure 1}

Steps in the simulation and analyses.

(A) Steps in the simulation (solid lines) and analyzed parameters (dashed lines) of the traditional paradigm. Test scores and a selection variable (sv) are affected by true $g(\mathrm{t} g$ ) but the effect is attenuated by disturbance that varies in magnitude between individuals. Amount of variance in the test scores explained by the first un-rotated principal component (ev) is calculated separately in the subgroups defined by the selection variable. Note: 14 test scores, besides the selection variable, were used in the actual simulation. (B) Illustration of the analyses when evaluating more contemporary criteria of SLODR. Test scores are regressed on either one single common $g$ factor (blue part) or on one linear and one quadratic $g$ factor (blue + red part). In the latter case, factor loadings for the quadratic factor are constrained to equal the square of the corresponding linear factor loadings. Note: 15 test scores were used in the actual simulation.
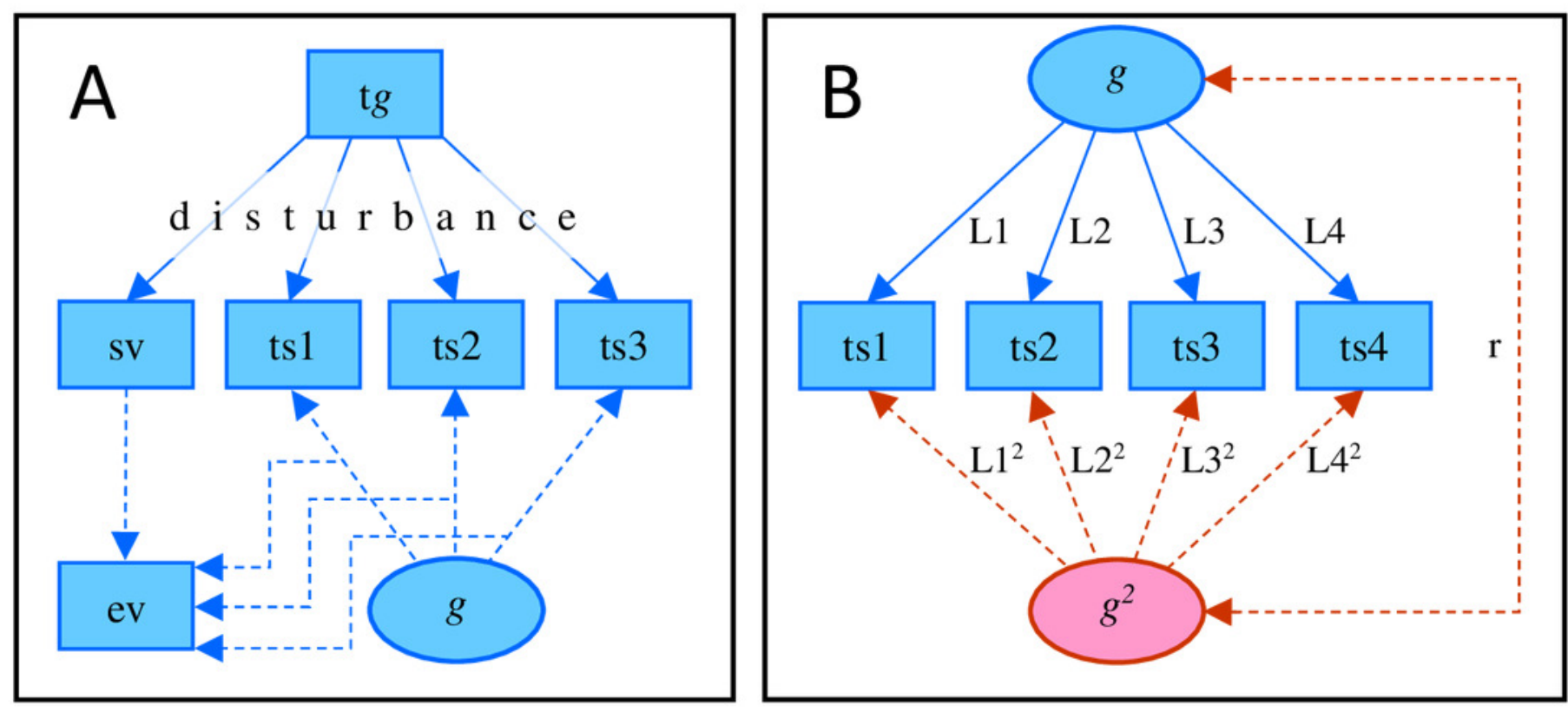


\section{Figure 2}

Explained variance as a function of mean intelligence.

Amount of variance in 14 tests explained by the first un-rotated principal component as a function of the mean value on the selection variable in the subgroups when the disturbance variable is either negatively, normally, or positively distributed or if there is no effect of disturbance on test scores. Separately for three different degrees of correlation between $g$ and the selection variable (before disturbance), namely (A) 0.1 , (B) 0.5 , and (C) 0.9 . The subgroups always have a maximum range of 20 points on the selection variable. 20 simulations (adjoined by a line) for each distribution of the disturbance variable in each panel and with $N=10,000$ in each.
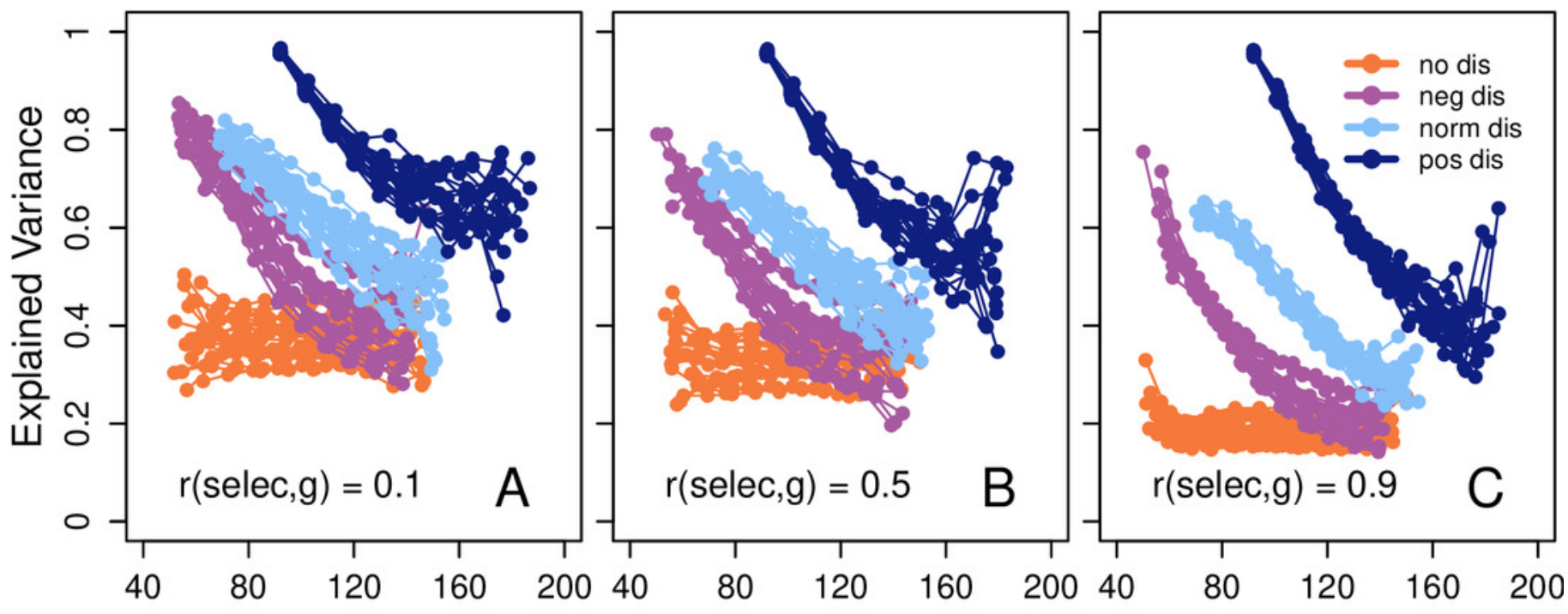

Mean on Selection Variable 


\section{Figure 3}

Illustration of the effect of disturbance.

Illustration of the effect of a negatively skewed disturbance variable. (A) A perfectly normally distributed true $g$ has (B) a weak linear association with the true score on one of the fifteen tests. When test scores are affected by a negatively skewed disturbance variable, we observe (C) a negatively skewed estimated $g$ that has (D) a quadratic association with the observed test score. We also observe heteroscedastic residuals that increase with increased $g(\mathrm{D}) . N=10,000$ in each panel, black line = best fitting linear association, red line $=$ best fitting quadratic association.
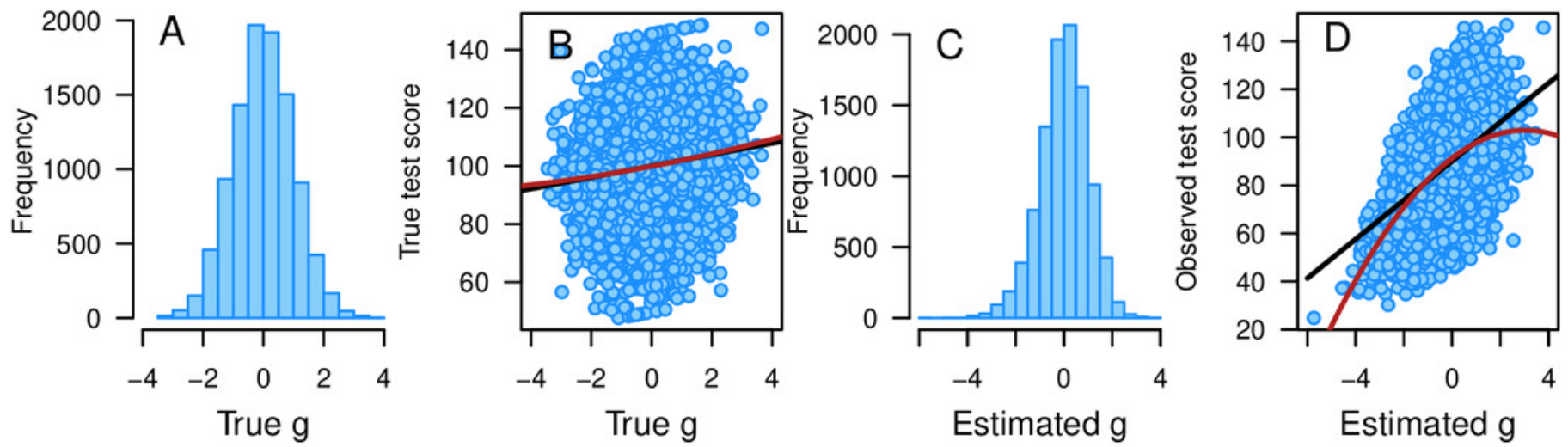


\section{Table $\mathbf{1}$ (on next page)}

Fulfillment of contemporary SLODR criteria.

Proportions of a significantly skewed common factor $(g)$, correlation between $g$ and residual variance, quadratic factor loadings, correlation between $g$ and the quadratic term $\left(g^{2}\right)$, and fulfilment of all crucial criteria as functions of the distribution of the disturbance variable. 
Table 1. Proportions of a significantly skewed common factor $(g)$, correlation between $g$ and residual variance, quadratic factor loadings, correlation between $g$ and the quadratic term $\left(g^{2}\right)$, and fulfilment of all crucial criteria as functions of the distribution of the disturbance variable.

\begin{tabular}{|c|c|c|c|c|}
\hline \multirow[b]{2}{*}{ Criteria } & \multicolumn{4}{|c|}{ Disturbance } \\
\hline & No & Negative & Normal & Positive \\
\hline Positive skew & 0.030 & 0.000 & 1.000 & 1.000 \\
\hline Negative skew & 0.027 & 1.000 & 0.000 & 0.000 \\
\hline$r(g, \text { res. })^{\mathrm{a}}>0$ & 0.021 & 1.000 & 1.000 & 1.000 \\
\hline$r(g, \text { res. })^{\mathrm{a}}<0$ & 0.032 & 0.000 & 0.000 & 0.000 \\
\hline$p\left(g^{2}\right)^{\mathbf{b}}<0.05$ & 0.031 & 0.997 & 0.998 & 0.945 \\
\hline$r\left(g, g^{2}\right)^{\mathrm{c}}>0$ & 0.010 & 0.000 & 0.000 & 0.000 \\
\hline$r\left(g, g^{2}\right)^{\mathrm{c}}<0$ & 0.012 & 0.811 & 0.974 & 0.940 \\
\hline $\mathrm{All}^{\mathrm{d}}$ & 0.000 & 0.811 & 0.000 & 0.000 \\
\hline
\end{tabular}

Note: 1000 simulations in each column, ${ }^{\mathrm{a}}$ correlation between $g$ and the logarithm of residual variance, ${ }^{\mathrm{b}}$ significance when adding a quadratic term to the factor model, ${ }^{\mathrm{c}}$ correlation between $g$ and the quadratic term, ${ }^{\mathrm{d}}$ fulfilment of all crucial criteria (in bold).

2 